\title{
Risk of Diabetes Mellitus in the Occurrence of Acute Ischemic Stroke at a Tertiary Care Hospital in Bangladesh
}

\author{
Nazia Sharmin 1,", Nasima Sultana ${ }^{2}$, Hafizur Rahman², Taniza Rahman ${ }^{3}$, Sanjella Nahreen Chowdhury ${ }^{4}$, \\ Md. Abdullah Yusuf ${ }^{5}$, Zakiur Rahman ${ }^{6}$
}

\author{
${ }^{1}$ Department of Biochemistry, Monno Medical College, Bangladesh \\ ${ }^{2}$ Department of Biochemistry, Dhaka Medical College, Bangladesh \\ ${ }^{3}$ Department of Biochemistry, Ashian Medical College, Bangladesh \\ ${ }^{4}$ Department of Biochemistry, Universal Medical College, Bangladesh \\ ${ }^{5}$ Department of Microbiology, National Institute of Neurosciences \& Hospital, Bangladesh \\ ${ }^{6}$ Department of Microbiology, Monno Medical College, Bangladesh
}

Copyright $\subset 2016$ by authors, all rights reserved. Authors agree that this article remains permanently open access under the terms of the Creative Commons Attribution License 4.0 International License

\begin{abstract}
Background: Acute ischemic stroke more frequently occurs in diabetic patients. Objective: The purpose of the present study was to observe the risk of diabetes mellitus in the occurrence of acute ischemic stroke in Bangladesh. Methodology: This case control study was carried out in the Department of Biochemistry at Dhaka Medical College, Dhaka, Bangladesh from January 2014 to December 2014 for a period of one (01) year. The case group consisted of male and female patients (age range 18-65 years) presenting with ischemic stroke. Age and sex matched healthy individuals were selected as the control group. History of diabetes of both cases and controls were recorded and blood sample was collected from both case and control after overnight fasting for the estimation of blood sugar. Results: A total number of 100 study subjects were taken of which 50 subjects presented with acute ischemic stroke were considered as case and the remaining 50 healthy subjects were taken as control. Diabetes mellitus was more commonly reported in case group $(46.0 \%)$ than control group $(6.0 \%)$ and the difference was found statistically significant $(\mathrm{p}=0.001)$. The risk estimation was calculated at 13.34 OR (95\% C.I. 3.66 to 48.62). Conclusions: The findings of this study conclude that diabetes mellitus was found to be significantly associated with acute ischemic stroke.
\end{abstract}

Keywords Acute Ischemic Stroke, Diabetes Mellitus, Risk Factors, Bangladesh

\section{Introduction}

Acute ischemic stroke is a globally increasing health problem. Stroke is, after cardiovascular disease and cancer, the $3^{\text {rd }}$ leading cause of death in the United States and a leading cause of serious, long term disability [1]. Stroke related annual cost is over $\$ 72$ billion [2]. An epidemiological study shows $85 \%$ of these strokes are ischemic [3]. Each year, approximately 795,000 people in the US experience new $(610,000$ people) or recurrent $(185,000)$ stroke [4]. About 4.4 million people die of stroke globally each year, of which almost three million are from developing countries [5]. In Bangladesh, stroke has been ranked as the third leading cause of death after coronary heart disease and infectious diseases such as influenza and pneumonia. The reported prevalence of stroke in Bangladesh is $0.3 \%$. The mortality rate of stroke increased $6.00 \%$ to $8.57 \%$ from 2006 to 2011 with an age-adjusted mortality rate of 108.31 per 100,000 people. The World Health Organization (WHO) ranks mortality due to stroke in Bangladesh as number 84 in the world [6]. Stroke is a medical emergency and can cause permanent neurological damage or death. Traditional risk factors for stroke include old age, high blood pressure, diabetes mellitus, hypercholesterolemia, previous history of stroke or TIA, obesity and dietary factors, atrial fibrillation and cigarette smoking [7].

Diabetes mellitus is considered as an important risk factor for stroke. In a study it has been indicated that even mild hyperglycemia in the acute infraction period is associated with poor outcome [7]. In Finish middle-aged population, diabetes mellitus was the strongest risk factor for death from stroke both in men and women [8]. Hyperglycemia may be directly toxic to the ischemic brain. Although, the mechanism is not fully understood, accumulation of lactate and intracellular acidosis in the ischemic brain produced through anaerobic cerebral glucose metabolism may contribute.

Most of data is from developed countries. Very little data are available in our country regarding this topic. Therefore, we assessed the association of diabetes mellitus in acute 
ischemic stroke cases of Bangladesh.

\section{Methodology}

This case control study was carried out in the Department of Biochemistry at Dhaka Medical College, Dhaka, Bangladesh from January 2014 to December 2014. Dhaka Medical College is a tertiary care teaching hospital with the capacity of 1000 beds. This is the oldest hospital in Bangladesh which has been situated in the heart of the Dhaka city. Patients from whole country have been referred to this hospital. Therefore, the participants was represented the Dhaka city as well as the country scenario. The patients were recruited by consecutively and purposive sampling method was used after fulfilling the inclusion and exclusion criteria. Ethical clearance from the concerned departments and authorities was taken. The written consent was taken from all the participants. The ischemic stroke patients were considered as case and the age and sex matched healthy volunteers were taken as control. Cases were the patients who clinically suffered from ischemic stroke confirmed by computerized tomography (CT) scan of brain attending in the Medicine unit of DMCH during the study period. A preformed data collection sheet was used to collect information regarding age, sex, BMI and fasting plasma glucose. Blood sample was collected from both case and control after overnight fasting for the estimation of blood sugar. Then, mean values of the quantitative variables were compared between case and control by Student's t-test and expressed as mean $\pm \mathrm{SD}$. Categorical variables were analyzed by using Chi square test. Finally, results were analyzed statistically in SPSS version 20. For all statistical analysis, $\mathrm{p}$ value $<0.05$ was considered as significant.

\section{Results}

A total number of 100 subjects were recruited for this study of which 50 were considered as cases and the remaining 50 were taken as healthy controls.

Table 1. Demographic characteristics of the subjects

\begin{tabular}{|cccc|}
\hline Variables & $\begin{array}{c}\text { Case } \\
(\mathrm{n}=50) \\
(\text { mean+SD) }\end{array}$ & $\begin{array}{c}\text { Control } \\
(\mathrm{n}=50) \\
(\text { mean+SD) }\end{array}$ & p value \\
\hline Age (years) & $56.54 \pm 12.18$ & $53.34 \pm 7.98$ & $0.124^{*}$ \\
BMI (Kg/m2) & $27.25 \pm 3.25$ & $25.38 \pm 2.57$ & $0.002^{*}$ \\
Sex & & & \\
Male & $32(64 \%)$ & $28(56 \%)$ & $0.414^{*}$ \\
Female & $18(36 \%)$ & $22(44 \%)$ & $*$ \\
\hline
\end{tabular}

*Student's $\mathrm{t}$ test was done to measure the level of significance; $* *$ Chi square test was done to measure the level of significance; Male: Female ratio in Case: 1.8:1; Male: Female ratio in Control: 1.3:1.

In this study, the mean $\pm \mathrm{SD}$ of age of case and control were $56.54 \pm 12.18$ and $53.34 \pm 7.98$ respectively. There was no statistically significant difference of mean age between groups $(p=0.124)$. Mean \pm SD of BMI was significantly higher in cases than that of controls $(\mathrm{p}=0.002)$ which were $27.25 \pm 3.25$ and $25.38 \pm 2.57 \mathrm{~kg} / \mathrm{m}^{2}$ respectively and table-I also showed that the study subjects were sex matched, the difference between case and control was not statistically significant $(\mathrm{p}=0.414)$. (Table-1)

Table 2. Distribution of study subjects by diabetes mellitus

\begin{tabular}{cccc}
\hline $\begin{array}{c}\text { Diabetes } \\
\text { mellitus }\end{array}$ & $\begin{array}{c}\text { Case } \\
(\text { Mean } \pm \text { SD) }\end{array}$ & $\begin{array}{c}\text { Control } \\
(\text { Mean } \pm \text { SD) }\end{array}$ & P value \\
\hline Present & $23(46.0)$ & $3(6.0)$ & \\
Absent & $27(54.0)$ & $47(94.0)$ & $0.001^{*}$ \\
Total & $\mathbf{5 0 ( 1 0 0 \% )}$ & $\mathbf{5 0}(\mathbf{1 0 0 \%})$ & \\
\hline
\end{tabular}

*Chi square test was done to measure the level of significance

In this study, $46 \%$ patients of case group and $6 \%$ respondents of control group had diabetes mellitus. Statistically significant difference was found between two groups regarding the presence of $\mathrm{DM}(\mathrm{p}=0.001)$. Here, $\mathrm{OR}=$ 13.34 (95\% C. I. 3.66 to 48.62$)$, Risk of ischemic stroke is 13.34 times more in diabetic subjects than that of non-diabetic subjects. (Table-2)

Table 3. Mean difference of FPG in case and control

\begin{tabular}{|cccc|}
\hline $\begin{array}{c}\text { Parameter } \\
(\mathrm{mmol} / \mathrm{L})\end{array}$ & $\begin{array}{c}\text { Case } \\
(\mathrm{mean} \pm \mathrm{SD})\end{array}$ & $\begin{array}{c}\text { Control } \\
(\mathrm{mean} \pm \mathrm{SD})\end{array}$ & P value \\
\hline $\mathrm{FPG}$ & $6.24 \pm 1.38$ & $4.74 \pm 0.80$ & $0.001^{*}$ \\
\hline
\end{tabular}

*Student's $t$ test was done to measure the level of significance; Significance $=(\mathrm{p}<0.05)$

The mean $\pm \mathrm{SD}$ of fasting plasma glucose (FPG) of the study subjects were recorded. FPG was significantly higher $(p=0.001)$ in cases than that of controls which were $6.24 \pm$ 1.38 and $4.74 \pm 0.80 \mathrm{mmol} / \mathrm{L}$ respectively (Table-3).

\section{Discussion}

In the present study an association of diabetes mellitus with acute ischemic stroke has been studied in a Bangladeshi population at a tertiary care hospital. In this study, the mean age \pm SD of case and control were $56.54 \pm 12.18$ years and $53.34 \pm 7.98$ years respectively. In this study the mean age of the case and control groups are nearly similar which indicates that the two groups are in nearest age with more or less similar SD. Furthermore the SD is also narrow. Thus this shows the two groups study population is age matched.

Among the study subjects there were $64 \%$ male and 36\% female in case group and $56 \%$ male and $44 \%$ female in control group. Male: Female ratio in Case: 1.8:1; Male: Female ratio in Control: 1.3:1; the sex of case and control were matched. In most age groups except older adults, stroke is more common in men than women. Stroke incidence rates are 1.25 times greater in men. But because of longer life span of women, more women than men die of stroke each year [9]. In Bangladesh, females in comparison to males are more neglected due to low socioeconomic condition. Therefore, very few number of female stroke patients can reached to the hospital for better outcome than male. 
Diabetes mellitus is an important risk factor for stroke. Uncontrolled diabetes causes premature atherosclerosis resulting cerebral thrombosis and ischemic stroke. Diabetes also causes endothelial proliferation and thickening of the basement membrane in the small blood vessel. Type $2 \mathrm{DM}$ in the elderly is associated with increased incidence of vascular disease, particularly, atherosclerosis of large blood vessels [7]. In this study, $46 \%$ patients of case group and $6 \%$ respondents of control group had DM. Statistical analysis showed significant difference between the two groups in term of presence of diabetes mellitus ( $\mathrm{p}=0.001)$. A hospital based case control study was done in Bangladesh with a sample size of 60 . In that study it was found $33.33 \%$ of cases and $10 \%$ of controls were diabetic [8].

The study showed mean \pm SD of FPG in cases was $6.24 \pm 1.38 \mathrm{mmol} / \mathrm{L}$ and in controls was $4.74 \pm 0.80 \mathrm{mmol} / \mathrm{L}$ respectively. The difference between them was statistically significant $(p=0.001)$. This result is consistent with the study result done by Abu-Odah, et al. [10]. They found that the stroke victims had higher fasting plasma glucose level $(133.57 \mathrm{mg} / \mathrm{dl}$ vs. $100.90 \mathrm{mg} / \mathrm{dl})$ than the control group.

\section{Conclusions}

In conclusion, diabetes mellitus was found to be significantly associated with acute ischemic stroke. So, this study suggests that diabetic subjects should keep a keen eye on their blood sugar level and if needed life style modification and weight reduction could be done.

\section{REFERENCES}

[1] GA. Donnan, M. Fisher, M. Macleod, SM. Davis. Stroke, Lancet, Vol. 371, 1612-1623, 2008.

[2] EC. Jauch. Ischemic stroke, 2015. Updated: Nov 23, 2015, Available online from: http://emedicine.medscape.com/articl e/1916852-overview\#a0156

[3] DB. Matcher, GW. Divine, A. Heyman, JR. Feussner. The influence of hyperglycemia on outcome of cerebral infarction, Annals of Internal Medicines, Vol. 117, 449-56, 1992.

[4] VL. Roger, AS. Go, DM. Lloyd-Jones, EJ. Benjamin, JD. Berry, WB. Borden. Heart disease and stroke statistics-2012 update: a report from the American Heart Association, Circulation, Vol. 125, No.1, $2^{2}-\mathrm{e}^{220}, 2012$.

[5] J. Tuomilehto, D. Rastenytė,P. Jousilahti, C. Sarti, E. Vartiainen. Diabetes Mellitus as a Risk Factor for Death From Stroke Prospective Study of the Middle-aged Finnish Population, Stroke, Vol. 27, 210-215, 1996.

[6] MN. Islam, M. Moniruzzaman, MI. Khalil, R. Basri, MK. Alam, KW. Loo, SH. Gan. Burden of stroke in Bangladesh, International Journal of Stroke, Vol. 8, No. 3, 211-13, 2013.

[7] DJ. Messier, N. Awad, M. Gayvaon.The relationships between atherosclerosis, heart diseases, type 2 diabetes and dementia, Neurological Research, Vol. 25, 567-572, 2004.s

[8] K. Kirtania, N. Sultana, MZ. Hossain, S. Ahmed, A. Khatun. Association of diabetes mellitus and smoking with ischemic stroke, Journal of Dhaka Medical College, Vol. 20, No. 1, 12-14, 2011.

[9] RL. Sacco, EJ. Benjamin, JP. Broderick, M. Dyken, JD. Easton, WM. Feinberg, LB. Goldstein, PB. Gorelick, G. Howard, SJ. Kittner, T. Manolio, JP. Whisnant, PA. Wolf. Risk factors: stroke, Stroke, American Stroke Association Journals, Vol. 28, 1507-1517, 1997.

[10] H. Abu-Odah, Y. Abed, B. Abu-Hamad. Risk Factors of Stroke in Patients Admitted in European Gaza Hospital, Gaza Strip: A case Control Study in Medical Unit Setting, Journal of Neurological Disorder- Stroke, Vol. 2, No.4, 1-5, 2014. 